\title{
The Neumann Problem for the 2-D Helmholtz Equation in a Multiply Connected Domain with Cuts
}

\author{
P. A. Krutitskii
}

\begin{abstract}
The Neumann problem for the dissipative Helmholtz equation in a connected plane region with cuts is studied. The existence of classical solution is proved by potential theory. The problem is reduced to a Fredholm equation of the second kind, which is uniquely solvable. It means that the solution can be computed by standard codes.
\end{abstract}

Keywords: Helmholtz equation, boundary value problems, cracks, screens, wings AMS subject classification: 73 D 25, 76 Q 05, 78 A 45, 45 E 05, 35 J 05, 35 J 25, 31 A 25

\section{Introduction}

The potential theory and the theory of boundary integral equations are the basic tools for studies of boundary value problems in different fields of applied mathematics. The majority of papers deals with problems in domains bounded by closed surfaces. Modern researches are mostly devoted to the generalized solvability in Sobolev and Besov spaces and to the extension of classical results to the case of Lipshitz boundaries. Recent advances in problems for the Laplace equation, wave propagation and the elastisity theory are presented in $[5,11,12,15,18,19,23)$ (see also the references in these papers).

Another direction of modern research $[1,2,4,7-10,17,18,21,22,24]$ deals with boundary value problems in the exterior of open surfaces. The open surfaces model cracks, screens or wings in physical problems.

It is very natural to join the two kinds of mentioned problems and to consider problems in general domains with boundaries containing both closed and open surfaces. Similar problems were not treated before even in the classical formulation for the 2dimensional Helmholtz equation. This situation can be explained by difficulties in the analysis of the boundary integral equation, which appear in these problems. Indeed, using classical single and double layer potentials in the 2-dimensional Neumann problem we arrive at a hypersingular integral equation on the open curves and at an equation of the second kind with compact integral operator on the closed curves. Clearly, the equation on the whole boundary is not classical and its analysis is quite complicated.

P. A. Krutitskii: Moscow State University, Department of Mathematics, Faculty of Physics, Moscow 119899, Russia 
In the present paper we suggest another way to study the Neumann problem for the 2-dimensional Helmholtz equation in a domain bounded by closed and open curves (cuts). With the help of the non-classical angular potential we obtain an integral equation, which contains Cauchy singular integrals on the open curves and compact integral operators on the closed curves. Besides, we must satisfy additional integral conditions arising from the nature of the angular potential. The solvability of the system of integral equations is proved in appropriate weighted Banach spaces. In doing so, the system is reduced to a Fredholm equation of the second kind on the whole boundary. The obtained Fredholm equation is uniquely solvable in the corresponding Banach space. Similar way has not been used before even in those cases, when the boundary contains only open curves or surfaces. For example, in $[1,4,9,10,17,18,20,24]$ the solvability of a boundary integral equation of the 1st kind has been proved directly, without reduction to a uniquely solvable Fredholm equation of the second kind, while such an equation is very useful for practical purposes, because it can be computed by standard codes.

Thus, the present note is an attempt to consider the Neumann problem for the Helmholtz equation in a 2 -dimensional external domain bounded by closed curves and containing cuts. Domains bounded by closed curves and exterior of cuts in a plane are particular cases of our problem. Our approach holds for both internal and external domains.

\section{Formulation of the problem}

In the plane $x=\left(x_{1}, x_{2}\right) \in \mathbb{R}^{2}$ we consider the multiply connected domain bounded by simple open curves $\Gamma_{1}^{1}, \ldots, \Gamma_{N_{1}}^{1} \in C^{2, \lambda}$ and simple closed curves $\Gamma_{1}^{2}, \ldots, \Gamma_{N_{2}}^{2} \in C^{1, \lambda} \quad(\lambda \in$ $(0,1])$, so that the curves do not have common points. We will consider both the case of an external domain and the case of an internal domain, when the curve $\Gamma_{1}^{2}$ encloses all other. We put

$$
\Gamma^{1}=\bigcup_{n=1}^{N_{1}} \Gamma_{n}^{1}, \quad \Gamma^{2}=\bigcup_{n=1}^{N_{2}} \Gamma_{n}^{2}, \quad \Gamma=\Gamma^{1} \cup \Gamma^{2} .
$$

The connected domain bounded by $\Gamma^{2}$ will be called $\mathcal{D}$. We assume that each curve $\Gamma_{n}^{k}\left(n=1, \ldots, N_{k} ; k=1,2\right)$ is parametricized by the arc length $s$ :

$$
\Gamma_{n}^{k}=\left\{x: x(s)=\left(x_{1}(s), x_{2}(s)\right) \text { for } s \in\left[a_{n}^{k}, b_{n}^{k}\right]\right\}
$$

so that

$$
a_{1}^{1}<b_{1}^{1}<\ldots<a_{N_{1}}^{1}<b_{N_{1}}^{1}<a_{1}^{2}<b_{1}^{2}<\ldots<a_{N_{2}}^{2}<b_{N_{2}}^{2} .
$$

and the domain $\mathcal{D}$ is to the right when the parameter $s$ increases on $\Gamma_{n}^{2}$. Therefore points $x \in \Gamma$ and values of the parameter $s$ are in one-to-one correspondence exept $a_{n}^{2}$ and $b_{n}^{2}$, which correspond to the same point $x$ for $n=1, \ldots, N_{2}$. Below the sets of the intervals on the $O s$ axis

$$
\bigcup_{n=1}^{N_{1}}\left[a_{n}^{1}, b_{n}^{1}\right], \quad \bigcup_{n=1}^{N_{2}}\left[a_{n}^{2}, b_{n}^{2}\right], \quad \bigcup_{k=1}^{2} \bigcup_{n=1}^{N_{k}}\left[a_{n}^{k}, b_{n}^{k}\right]
$$


will be denoted by $\Gamma^{1}, \Gamma^{2}$ and $\Gamma$ also.

We consider $\Gamma^{1}$ as a set of cuts. The side of $\Gamma^{1}$, which is on the left when the parameter $s$ increases, will be denoted by $\left(\Gamma^{1}\right)^{+}$and the opposite side will be denoted by $\left(\Gamma^{1}\right)^{-}$.

We put

$$
C^{0, r}\left(\Gamma_{n}^{2}\right)=\left\{\mathcal{F} \in C^{0, r}\left[a_{n}^{2}, b_{n}^{2}\right] \text { with } \mathcal{F}\left(a_{n}^{2}\right)=\mathcal{F}\left(b_{n}^{2}\right)\right\} \quad(r \in[0,1])
$$

and

$$
C^{0, r}\left(\Gamma^{2}\right)=\bigcap_{n=1}^{N_{2}} C^{0, r}\left(\Gamma_{n}^{2}\right) .
$$

The tangent vector to $\Gamma$ at the point $x(s)$ we denote by $\tau_{x}=(\cos \alpha(s), \sin \alpha(s))$, where $\cos \alpha(s)=x_{1}^{\prime}(s)$ and $\sin \alpha(s)=x_{2}^{\prime}(s)$. Let $\mathbf{n}_{x}=(\sin \alpha(s),-\cos \alpha(s))$ be a normal vector to $\Gamma$ at $x(s)$. The direction of $\mathbf{n}_{x}$ is chosen such that it will coincide with the direction of $\tau_{x}$ if $\mathbf{n}_{x}$ is rotated anticlockwise through an angle of $\frac{\pi}{2}$.

We say, that the function $u=u(x)$ belongs to the smoothness class $\mathbf{K}$ if the following conditions are fulfilled:

1) $u \in C^{0}\left(\overline{\mathcal{D} \backslash \Gamma^{1}}\right) \cap C^{2}\left(\mathcal{D} \backslash \Gamma^{1}\right)$.

2) $\nabla u \in C^{0}\left(\overline{\mathcal{D} \backslash \Gamma^{1}} \backslash X\right)$, where $X$ is a point-set, consisting of the end-points of $\Gamma^{1}$, i.e.

$$
X=\bigcup_{n=1}^{N_{1}}\left(x\left(a_{n}^{1}\right) \cup x\left(b_{n}^{1}\right)\right)
$$

3) In the neighbourhood of any point $x(d) \in X$, for some constants $\mathcal{C}>0$ and $\epsilon>-1$, the inequality

$$
|\nabla u| \leq \mathcal{C}|x-x(d)|^{\epsilon}
$$

holds where $x \rightarrow x(d)$ and $d=a_{n}^{1}$ or $d=b_{n}^{1} \quad\left(n=1, \ldots, N_{1}\right)$.

Let us formulate the Neumann problem for the dissipative Helmholtz equation in the domain $\mathcal{D} \backslash \Gamma^{1}$.

Problem (U). To find a function $u=u(x)$ of the class $K$ which satisfies the Helmholtz equation

$$
u_{x_{1} x_{1}}(x)+u_{x_{2} x_{2}}(x)+\beta^{2} u(x)=0 \quad\left(x \in \mathcal{D} \backslash \Gamma^{1}\right)
$$

where $\beta=$ const and $\operatorname{Im} \beta>0$, and satisfies the boundary conditions

$$
\begin{gathered}
\left.\frac{\partial u(x)}{\partial \mathbf{n}_{x}}\right|_{x(s) \in\left(\Gamma^{1}\right)^{+}}=F^{+}(s) \\
\left.\frac{\partial u(x)}{\partial \mathbf{n}_{x}}\right|_{x(s) \in\left(\Gamma^{1}\right)^{-}}=F^{-}(s) \\
\left.\frac{\partial u(x)}{\partial \mathbf{n}_{x}}\right|_{x(s) \in \Gamma^{2}}=F(s) .
\end{gathered}
$$


If $\mathcal{D}$ is an external domain, then we add at infinity the condition

$$
u=o\left(|x|^{-\frac{1}{2}}\right), \quad|\nabla u(x)|=o\left(|x|^{-\frac{1}{2}}\right), \quad|x|=\sqrt{x_{1}^{2}+x_{2}^{2}} \rightarrow \infty .
$$

All conditions of the problem $\mathbf{U}$ must be satisfied in the classical sense.

On the basis of the energy equalities we can easily prove the following assertion.

Theorem 1. If $\Gamma^{1} \in C^{2, \lambda}$ and $\Gamma^{2} \in C^{1, \lambda}(\lambda \in(0,1])$, then the problem $\mathrm{U}$ has at most one solution.

The theorem holds for both the internal and external domain $\mathcal{D}$.

\section{Integral equations at the boundary}

Below we assume that the functions $F^{+}=F^{+}(s), F^{-}=F^{-}(s)$ and $F=F(s)$ in (2)b belong to the following spaces:

$$
F^{+}, F^{-} \in C^{0, \lambda}\left(\Gamma^{1}\right) \quad \text { and } \quad F \in C^{0, \lambda}\left(\Gamma^{2}\right) \quad(\lambda \in(0,1]) .
$$

If $\mathcal{B}_{1}\left(\Gamma^{1}\right)$ and $\mathcal{B}_{2}\left(\Gamma^{2}\right)$ are Banach spaces of functions given on $\Gamma^{1}$ and $\Gamma^{2}$, respectively, then for functions given on $\Gamma$ we introduce the Banach space $\mathcal{B}_{1}\left(\Gamma^{1}\right) \cap \mathcal{B}_{2}\left(\Gamma^{2}\right)$ with the norm $\|\cdot\|_{B_{1}\left(\Gamma^{2}\right) \cap B_{2}\left(\Gamma^{2}\right)}=\|\cdot\|_{B_{1}\left(\Gamma^{2}\right)}+\|\cdot\|_{B_{2}\left(\Gamma^{2}\right)}$.

We consider the angular potential $w_{1}[\mu]=w_{1}[\mu](x)$ from $[13,14]$ for the equation $(2)_{a}$ on $\Gamma^{1}$ given by

$$
w_{1}[\mu](x)=\frac{i}{4} \int_{\Gamma^{1}} \mu(\sigma) V(x, \sigma) d \sigma .
$$

The kernel $V$ is defined on each curve $\Gamma_{n}^{1}\left(n=1, \ldots, N_{1}\right)$ by the formula

$$
V(x, \sigma)=\int_{a_{n}^{1}}^{\sigma} \frac{\partial}{\partial \mathbf{n}_{y}} \mathcal{H}_{0}^{(1)}(\beta|x-y(\xi)|) d \xi \quad\left(\sigma \in\left[a_{n}^{1}, b_{n}^{1}\right]\right)
$$

where $\mathcal{H}_{0}^{(1)}$ is the Hankel function of the first kind given by

$$
\mathcal{H}_{0}^{(1)}(z)=\frac{\sqrt{2} \exp \left(i z-i \frac{\pi}{4}\right)}{\pi \sqrt{z}} \int_{0}^{\infty} \exp (-t) t^{-\frac{1}{2}}\left(1+\frac{i t}{2 z}\right)^{-\frac{1}{2}} d t
$$

with $y(\xi)=\left(y_{1}(\xi), y_{2}(\xi)\right)$ and $|x-y(\xi)|=\sqrt{\left(x_{1}-y_{1}(\xi)\right)^{2}+\left(x_{2}-y_{2}(\xi)\right)^{2}}$.

Below we suppose that the function $\mu=\mu(x)$ belongs to the Banach space $C_{q}^{\omega}\left(\Gamma^{1}\right)$ $(\omega \in(0,1]), q \in[0,1))$ and satisfies the additional conditions

$$
\int_{a_{n}^{1}}^{b_{n}^{1}} \mu(\sigma) d \sigma=0 \quad\left(n=1, \ldots, N_{1}\right) .
$$


We say that $\mu \in C_{q}^{\omega}\left(\Gamma^{1}\right)$ if

$$
\mu(\cdot) \prod_{n=1}^{N_{1}}\left|\cdot-a_{n}^{1}\right|^{q}\left|\cdot-b_{n}^{1}\right|^{q} \in C^{0, w}\left(\Gamma^{1}\right)
$$

where $C^{0, \omega}\left(\Gamma^{1}\right)$ is the Hölder space with index $\omega$ and

$$
\|\mu\|_{C_{\sigma}^{\omega}\left(\Gamma^{1}\right)}=\left\|\mu(\cdot) \prod_{n=1}^{N_{1}}\left|\cdot-a_{n}^{1}\right|^{q}\left|\cdot-b_{n}^{1}\right|^{q}\right\|_{C^{0, \omega}\left(\Gamma^{1}\right)} .
$$

As shown in $[13,14]$, for such $\mu$ the angular potential $w_{1}[\mu](x)$ belongs to the class K. In particular, the inequality (1) holds with $\epsilon=-q$ if $q \in(0,1)$. Moreover, integrating $w_{1}[\mu](x)$ by parts and using (4) we express the angular potential in terms of a double layer potential

$$
w_{1}[\mu](x)=-\frac{i}{4} \int_{\Gamma^{1}} \rho(\sigma) \frac{\partial}{\partial \mathbf{n}_{\mathbf{y}}} \mathcal{H}_{0}^{(1)}(\beta|x-y(\sigma)|) d \sigma
$$

with the density

$$
\rho(\sigma)=\int_{a_{n}^{1}}^{\sigma} \mu(\xi) d \xi \quad\left(\sigma \in\left[a_{n}^{1}, b_{n}^{1}\right] ; n=1, \ldots, N_{1}\right)
$$

Consequently, $w_{1}[\mu](x)$ satisfies both the equation $(2)_{a}$ outside $\Gamma^{1}$ and the conditions at infinity $(2)_{c}$.

Let us construct a solution of the problem $U$. This solution can be obtained with the help of potential theory for the Helmholtz equation (2a). We seek a solution of the problem in the form

$$
W[\nu, \mu](x)=v_{1}[\nu](x)+w[\mu](x)
$$

where

$$
v_{1}[\nu](x)=\frac{i}{4} \int_{\Gamma^{1}} \nu(\sigma) \mathcal{H}_{0}^{(1)}(\beta|x-y(\sigma)|) d \sigma
$$

and

$$
w[\mu](x)=w_{1}[\mu](x)+w_{2}[\mu](x)
$$

with $w_{1}[\mu](x)$ given by (4), (6) and

$$
w_{2}[\mu](x)=\frac{i}{4} \int_{\Gamma^{2}} \mu(\sigma) \mathcal{H}_{0}^{(1)}(\beta|x-y(\sigma)|) d \sigma .
$$

By $\int_{\Gamma^{k}} \ldots d \sigma$ we mean

$$
\sum_{n=1}^{N_{k}} \int_{a_{n}^{k}}^{b_{n}^{k}} \ldots d \sigma
$$


We will look for $\nu=\nu(s)$ in the space $C^{0, \lambda}\left(\Gamma^{1}\right)$.

We will seek $\mu=\mu(s)$ from the Banach space $C_{q}^{\omega}\left(\Gamma^{1}\right) \cap C^{0, \frac{\lambda}{2}}\left(\Gamma^{2}\right)(\omega \in(0,1], q \in$ $[0,1))$ with the norm $\|\cdot\|_{C_{q}^{\omega}\left(\Gamma^{1}\right) \cap C^{0, \frac{\partial}{2}}\left(\Gamma^{2}\right)}=\|\cdot\|_{C_{q}^{\omega}\left(\Gamma^{1}\right)}+\|\cdot\|_{C^{0, \frac{1}{2}}\left(\Gamma^{2}\right)}$. Besides, $\mu$ must satisfy the conditions (5).

It follows from [13] that for such $\mu$ and $\nu$ the function (7) belongs to the class $\mathbf{K}$ and satisfies all conditions of the problem $U$ except the boundary condition $(2)_{b}$. In the case of the external domain $\mathcal{D}$ the function (7) satisfies the condition at infinity $(2)_{c}$.

To satisfy the boundary condition we put (7) into $(2)_{b}$, use the limit formulas for the angular potential from [13] and arrive for the densities $\mu$ and $\nu$ at the integral equations

$$
\begin{aligned}
& \pm \frac{1}{2} \nu(s)+\frac{i}{4} \int_{\Gamma^{1}} \nu(\sigma) \frac{\partial}{\partial \mathbf{n}_{x}} \mathcal{H}_{0}^{(1)}(\beta|x(s)-y(\sigma)|) d \sigma \\
& \quad-\frac{1}{2 \pi} \int_{\Gamma^{1}} \mu(\sigma) \frac{\sin \varphi_{0}(x(s), y(\sigma))}{|x(s)-y(\sigma)|} d \sigma \\
& \quad+\frac{i}{4} \int_{\Gamma^{1}} \mu(\sigma) \frac{\partial}{\partial \mathbf{n}_{x}} V_{0}(x(s), \sigma) d \sigma \\
& \quad+\frac{i}{4} \int_{\Gamma^{2}} \mu(\sigma) \frac{\partial}{\partial \mathbf{n}_{x}} \mathcal{H}_{0}^{(1)}(\beta|x(s)-y(\sigma)|) d \sigma=F^{ \pm}(s)
\end{aligned}
$$

and

$$
\begin{aligned}
\frac{i}{4} \int_{\Gamma^{1}} & \nu(\sigma) \frac{\partial}{\partial \mathbf{n}_{x}} \mathcal{H}_{0}^{(1)}(\beta|x(s)-y(\sigma)|) d \sigma \\
& -\frac{1}{2 \pi} \int_{\Gamma^{1}} \mu(\sigma) \frac{\sin \varphi_{0}(x(s), y(\sigma))}{|x(s)-y(\sigma)|} d \sigma \\
& +\frac{i}{4} \int_{\Gamma^{1}} \mu(\sigma) \frac{\partial}{\partial \mathbf{n}_{x}} V_{0}(x(s), \sigma) d \sigma-\frac{1}{2} \mu(s) \\
& +\frac{i}{4} \int_{\Gamma^{2}} \mu(\sigma) \frac{\partial}{\partial \mathbf{n}_{x}} \mathcal{H}_{0}^{(1)}(\beta|x(s)-y(\sigma)|) d \sigma=F(s)
\end{aligned}
$$

where

$$
V_{0}(x, \sigma)=\int_{a_{n}^{1}}^{\sigma} \frac{\partial}{\partial \mathbf{n}_{y}} h(\beta|x-y(\xi)|) d \xi \quad\left(\sigma \in\left[a_{n}^{1}, b_{n}^{1}\right] ; n=1,2, \ldots, N_{1}\right)
$$

and

$$
h(z)=\mathcal{H}_{0}^{(1)}(z)-\frac{2 i}{\pi} \ln \frac{z}{\beta} .
$$

By $\varphi_{0}(x, y)$ we denote the angle between the vector $\overrightarrow{x y}$ and the direction of the normal $\mathbf{n}_{\boldsymbol{x}}$. This angle is taken to be positive if it is measured anticlockwise from $\mathbf{n}_{\boldsymbol{x}}$ and negative if it is measured clockwise from $\mathbf{n}_{x}$. Besides, $\varphi_{0}(x, y)$ is continuous in $x, y \in \Gamma$ if $x \neq y$.

Equation $(9)_{a}$ is obtained as $x \rightarrow x(s) \in\left(\Gamma^{1}\right)^{ \pm}$and comprises two integral equations. The upper sign denotes the integral equation on $\left(\Gamma^{1}\right)^{+}$, the lower sign denotes the 
integral equation on $\left(\Gamma^{1}\right)^{-}$. In addition to the integral equations written above we have the conditions (5).

Subtracting the integral equations $(9)_{a}$ we find

$$
\nu(s)=F^{+}(s)-F^{-}(s) \quad \text { and } \quad \nu \in C^{0, \lambda}\left(\Gamma^{1}\right) .
$$

We note that $\nu$ is found completely and satisfies all required conditions. Hence, the potential $v_{1}[\nu]$ is found completely as well.

We introduce the function $f=f(s)$ on $\Gamma$ by the formula

$$
f(s)=F(s)-\frac{i}{4} \int_{\Gamma^{1}}\left(F^{+}(\sigma)-F^{-}(\sigma)\right) \frac{\partial}{\partial \mathbf{n}_{x}} \mathcal{H}_{0}^{(1)}(\beta|x(s)-y(\sigma)|) d \sigma \quad(s \in \Gamma)
$$

where

$$
F(s)=\frac{1}{2}\left(F^{+}(s)+F^{-}(s)\right) \quad\left(s \in \Gamma^{1}\right) .
$$

As shown in [14], $f \in C^{0, \lambda}\left(\Gamma^{1}\right)$. Hence, $f \in C^{0, \lambda}(\Gamma)$.

Adding the integral equations $(9)_{a}$ and taking into account $(9)_{b}$ we obtain for $\mu$ on $\Gamma$ the integral equation

$$
\begin{aligned}
& -\frac{1}{2 \pi} \int_{\Gamma^{1}} \mu(\sigma) \frac{\sin \varphi_{0}(x(s), y(\sigma))}{|x(s)-y(\sigma)|} d \sigma \\
& +\frac{i}{4} \int_{\Gamma^{1}} \mu(\sigma) \frac{\partial}{\partial \mathbf{n}_{x}} V_{0}(x(s), \sigma) d \sigma-\frac{1}{2} \delta(s) \mu(s) \quad(s \in \Gamma) \\
& +\frac{i}{4} \int_{\Gamma^{2}} \mu(\sigma) \frac{\partial}{\partial \mathbf{n}_{x}} \mathcal{H}_{0}^{(1)}(\beta|x(s)-y(\sigma)|) d \sigma=f(s)
\end{aligned}
$$

where $\delta(s)=0$ if $s \in \Gamma^{1}$ and $\delta(s)=1$ if $s \in \Gamma^{2}$, and where $f$ is given on $\Gamma$ by (11).

Let us show that any solution of equation (12), which is integrable on $\Gamma^{1}$ and continuous on $\Gamma^{2}$, belongs to $C^{0, \frac{\lambda}{2}}\left(\Gamma^{2}\right)$. Indeed, it follows from $[13,14]$ that if $s \in \Gamma^{2}$, then the kernel of the integral term in (12) can be expressed in the form

$$
\frac{I_{0}(s, \sigma)}{s-\sigma}+I_{1}(s, \sigma)
$$

where $I_{0} \in C^{0, \lambda}\left(\Gamma^{2} \times \Gamma\right)$ with $I_{0}(s, s)=0$ and $I_{1} \in C^{0, \frac{\lambda}{2}}\left(\Gamma^{2} \times \Gamma\right)$. From [16] we obtain

$$
\frac{I_{0}(s, \sigma)}{s-\sigma}+I_{1}(s, \sigma)=\frac{I_{2}(s, \sigma)}{|s-\sigma|^{1-\frac{\lambda}{2}}}+I_{1}(s, \sigma)
$$

where $I_{2} \in C^{0, \frac{\lambda}{2}}\left(\Gamma^{2} \times \Gamma\right)$. In accordance with [5], due to this representation, the integral term from (12) belongs to $C^{0, \frac{\lambda}{2}}\left(\Gamma^{2}\right)$. Since $f \in C^{0, \lambda}\left(\Gamma^{2}\right)$, the solution $\mu$ of equation (12) belongs to $C^{0, \frac{\lambda}{2}}\left(\Gamma^{2}\right)$. Thus, if $\mu$ is a solution of equations (5), (12) from the space $C_{q}^{\omega}\left(\Gamma^{1}\right) \cap C^{0}\left(\Gamma^{2}\right) \quad(\omega \in(0,1], q \in[0,1))$, then $\mu \in C_{q}^{\omega}\left(\Gamma^{1}\right) \cap C^{0, \frac{\lambda}{2}}\left(\Gamma^{2}\right)$ and the potential (7) satisfies all conditions of the problem $\mathbf{U}$.

The following theorem holds. 
Theorem 2. If $\Gamma^{1} \in C^{2, \lambda}$ and $\Gamma^{2} \in C^{1, \lambda}$, if conditions (3) hold and the system of equations (12), (5) has a solution $\mu=\mu(s)$ from the Banach space $C_{q}^{\omega}\left(\Gamma^{1}\right) \cap C^{0}\left(\Gamma^{2}\right)(\omega \in$ $(0,1], q \in[0,1))$, then a solution of the problem $\mathrm{U}$ is given by $(7)$, where $\nu=\nu(s)$ is defined in (10).

Below we look for $\mu$ in the Banach space $C_{q}^{w}\left(\Gamma^{1}\right) \cap C^{0}\left(\Gamma^{2}\right)$. If $s \in \Gamma^{2}$, then (12) is an equation of the second kind with compact integral operators. If $s \in \Gamma^{1}$, then (12) is a singular integral equation [5].

Our further treatment will be aimed to the proof of the solvability of the system (5), (12) in the Banach space $C_{q}^{\omega}\left(\Gamma^{1}\right) \cap C^{0}\left(\Gamma^{2}\right)$. Moreover, we reduce the system (5), (12) to a Fredholm equation of the second kind, which can be easily computed by classical methods.

Equation (12) on $\Gamma^{2}$ we rewrite in the form

$$
\mu(s)+\int_{\Gamma^{\prime}} \mu(\sigma) A_{2}(s, \sigma) d \sigma=-2 f(s) \quad\left(s \in \Gamma^{2}\right)
$$

where

$$
\begin{aligned}
A_{2}(s, \sigma) & =-\left\{\frac{i}{2}(1-\delta(\sigma)) \frac{\partial}{\partial \mathbf{n}_{x}} V(x(s), \sigma)+\frac{i}{2} \delta(\sigma) \frac{\partial}{\partial \mathbf{n}_{x}} \mathcal{H}_{0}^{(1)}(\beta|x(s)-y(\sigma)|)\right\} \\
& =\frac{I_{2}(s, \sigma)}{|s-\sigma|^{1-\frac{\lambda}{2}}}+I_{1}(s, \sigma) .
\end{aligned}
$$

Here $V$ is the kernel of the angular potential (4) and $I_{j} \in C^{0, \frac{\lambda}{2}}\left(\Gamma^{2} \times \Gamma\right)(j=1,2)$ as shown above.

It can be easily proved that

$$
\frac{\sin \varphi_{0}(x(s), y(\sigma))}{|x(s)-y(\sigma)|}-\frac{1}{\sigma-s} \in C^{0, \lambda}\left(\Gamma^{1} \times \Gamma^{1}\right)
$$

(see $[13,14]$ for details). Therefore we can rewrite (12) on $\Gamma^{1}$ in the form

$$
\frac{1}{\pi} \int_{\Gamma^{1}} \mu(\sigma) \frac{d \sigma}{\sigma-s}+\int_{\Gamma} \mu(\sigma) Y(s, \sigma) d \sigma=-2 f(s) \quad\left(s \in \Gamma^{1}\right)
$$

where

$$
\begin{aligned}
Y(s, \sigma)= & \left\{(1-\delta(\sigma))\left[\frac{1}{\pi}\left(\frac{\sin \varphi_{0}(x(s), y(\sigma))}{|x(s)-y(\sigma)|}-\frac{1}{\sigma-s}\right)-\frac{i}{2} \frac{\partial}{\partial \mathbf{n}_{x}} V_{0}(x(s), \sigma)\right]\right. \\
& \left.-\frac{i}{2} \delta(\sigma) \frac{\partial}{\partial \mathbf{n}_{x}} \mathcal{H}_{0}^{(1)}(\beta|x(s)-y(\sigma)|)\right\} \\
\in & C^{0, p_{0}}\left(\Gamma^{1} \times \Gamma\right)
\end{aligned}
$$

with $p_{0}=\lambda$ if $0<\lambda<1$ and $p_{0}=1-\epsilon_{0}$ for any $\epsilon_{0} \in(0,1)$ if $\lambda=1$. 


\section{The Fredholm integral equation and the solution of the problem}

Inverting the singular integral operator in (14) we arrive at the following integral equation of the second kind [16]:

$$
\begin{aligned}
\mu(s)+\frac{1}{Q_{1}(s)} \int_{\Gamma} \mu(\sigma) A_{0}(s, \sigma) d \sigma & +\frac{1}{Q_{1}(s)} \sum_{n=0}^{N_{1}-1} G_{n} s^{n} \quad\left(s \in \Gamma^{1}\right) \\
& =\frac{1}{Q_{1}(s)} \Phi_{0}(s)
\end{aligned}
$$

where

$$
\begin{aligned}
A_{0}(s, \sigma) & =-\frac{1}{\pi} \int_{\Gamma^{1}} \frac{Y(\xi, \sigma)}{\xi-s} Q_{1}(\xi) d \xi \\
Q_{1}(s) & =\prod_{n=1}^{N_{1}}\left|\sqrt{s-a_{n}^{1}} \sqrt{b_{n}^{1}-s}\right| \operatorname{sign}\left(s-a_{n}^{1}\right) \\
\Phi_{0}(s) & =\frac{1}{\pi} \int_{\Gamma^{1}} \frac{2 Q_{1}(\sigma) f(\sigma)}{\sigma-s} d \sigma
\end{aligned}
$$

and $G_{0}, \ldots, G_{N_{1}-1}$ are arbitrary constants. To derive equations for these constants we substitute $\mu$ from (15) into the conditions (5) and obtain

$$
\int_{\Gamma} \mu(\sigma) l_{n}(\sigma) d \sigma+\sum_{m=0}^{N_{1}-1} B_{n m} G_{m}=H_{n} \quad\left(n=1, \ldots, N_{1}\right)
$$

where

$$
\begin{aligned}
l_{n}(\sigma) & =-\int_{\Gamma_{n}^{1}} Q_{1}^{-1}(s) A_{0}(s, \sigma) d s \\
B_{n m} & =-\int_{\Gamma_{n}^{1}} Q_{1}^{-1}(s) s^{m} d s \\
H_{n} & =-\int_{\Gamma_{n}^{1}} Q_{1}^{-1}(s) \Phi_{0}(s) d s .
\end{aligned}
$$

By $B$ we denote the $\left(N_{1} \times N_{1}\right)$-matrix with the elements $B_{n m}$ from (17). As shown in [14], this matrix is invertible. The elements of the inverse matrix $B^{-1}$ will be called $\left(B^{-1}\right)_{n m}$. Inverting the matrix $B$ into (16) we express the constants $G_{0}, \ldots, G_{N_{1}-1}$ in terms of $\mu$ :

$$
G_{n}=\sum_{m=1}^{N_{1}}\left(B^{-1}\right)_{n m}\left[H_{m}-\int_{\Gamma} \mu(\sigma) l_{m}(\sigma) d \sigma\right] .
$$

We substitute $G_{n}$ into (15) and obtain the following integral equation for $\mu$ on $\Gamma^{1}$ :

$$
\mu(s)+\frac{1}{Q_{1}(s)} \int_{\Gamma} \mu(\sigma) A_{1}(s, \sigma) d \sigma=\frac{1}{Q_{1}(s)} \Phi_{1}(s) \quad\left(s \in \Gamma^{1}\right)
$$


where

$$
A_{1}(s, \sigma)=A_{0}(s, \sigma)-\sum_{n=0}^{N_{1}-1} s^{n} \sum_{m=1}^{N_{1}}\left(B^{-1}\right)_{n m} l_{m}(\sigma)
$$

and

$$
\Phi_{1}(s)=\Phi_{0}(s)-\sum_{n=0}^{N_{1}-1} s^{n} \sum_{m=1}^{N_{1}}\left(B^{-1}\right)_{n m} H_{m} .
$$

It can be shown using the properties of singular integrals $[6,16]$ that $\Phi_{0}=\Phi_{0}(s)$ and $A_{0}=A_{0}(s, \sigma) \quad\left(s \in \Gamma^{1}, \sigma \in \Gamma\right)$ are Hölder functions. Therefore, $\Phi_{1}=\Phi_{1}(s)$ and $A_{1}=A_{1}(s, \sigma)\left(s \in \Gamma^{1}, \sigma \in \Gamma\right)$ are also Hölder functions. Consequently, any solution of (18) belongs to $C_{\frac{1}{2}}^{\omega}\left(\Gamma^{1}\right)$ and below we look for $\mu$ on $\Gamma^{1}$ in this space.

We put

$$
Q(s)=(1-\delta(s)) Q_{1}(s)+\delta(s) \quad(s \in \Gamma) .
$$

Instead of $\mu \in C_{\frac{1}{2}}^{\omega}\left(\Gamma^{1}\right) \cap C^{0}\left(\Gamma^{2}\right)$ we introduce the new unknown function $\mu_{*}(s)=$ $\mu(s) Q(s)$, so that $\mu_{*} \in C^{0, \omega}\left(\Gamma^{1}\right) \cap C^{0}\left(\Gamma^{2}\right)$, and rewrite (13), (18) in the form of one equation

$$
\mu_{*}(s)+\int_{\Gamma} \mu_{*}(\sigma) Q^{-1}(\sigma) A(s, \sigma) d \sigma=\Phi(s) \quad(s \in \Gamma)
$$

where

$$
A(s, \sigma)=(1-\delta(s)) A_{1}(s, \sigma)+\delta(s) A_{2}(s, \sigma)
$$

and

$$
\Phi(s)=(1-\delta(s)) \Phi_{1}(s)-2 \delta(s) f(s) .
$$

Thus, the system of equations (12), (5) for $\mu=\mu(s)$ has been reduced to the equation (19) for the function $\mu_{*}=\mu_{*}(s)$. It is clear from our consideration that any solution of the equation (19) gives a solution of the system (12), (5).

As noted above, $\Phi_{1}=\Phi(s)$ and $A_{1}=A_{1}(s, \sigma)\left(s \in \Gamma^{1}, \sigma \in \Gamma\right)$ are Hölder functions. More precisely (see $[14,16]), \Phi_{1} \in C^{0, p}\left(\Gamma^{1}\right)$ for $p=\min \left\{\frac{1}{2}, \lambda\right\}$ and $A_{1}(\cdot, \sigma) \in C^{0, p}\left(\Gamma^{1}\right)$ uniformly with respect to $\sigma \in \Gamma$.

We arrive at the following assertion.

Lemma. If $\Gamma^{1} \in C^{2, \lambda}$ and $\Gamma^{2} \in C^{1, \lambda}(\lambda \in(0,1]), \Phi \in C^{0, p}\left(\Gamma^{1}\right) \cap C^{0}\left(\Gamma^{2}\right)$ for $p=$ $\min \left\{\lambda, \frac{1}{2}\right\}$, and if $\mu_{*} \in C^{0}(\Gamma)$ satisfies the equation (19), then $\mu_{*} \in C^{0, p}\left(\Gamma^{1}\right) \cap C^{0}\left(\Gamma^{2}\right)$.

The condition $\Phi \in C^{0, p}\left(\Gamma^{1}\right) \cap C^{0}\left(\Gamma^{2}\right)$ holds if $f \in C^{0, \lambda}(\Gamma)$. Hence below we will seek $\mu_{*}=\mu_{*}(s)$ from $C^{0}(\Gamma)$. Since $A_{1} \in C^{0}\left(\Gamma^{1} \times \Gamma\right)$ and due to the special representation for $A_{2}$ from (13), the integral operator from (19)

$$
\mathbf{A} \mu_{*}=\int_{\Gamma} \mu_{*}(\sigma) Q^{-1}(\sigma) A(s, \sigma) d \sigma
$$

is a compact operator mapping $C^{0}(\Gamma)$ into itself. Therefore, (19) is a Fredholm equation of the second kind in the Banach space $C^{0}(\Gamma)$. 
Let us show that the homogeneous equation (19) has only a trivial solution. Then, according to Fredholm's theorems, the inhomogeneous equation (19) has a unique solution for any right-hand side. We will prove this by a contradiction. Let $\mu_{*}^{0} \in C^{0}(\Gamma)$ be a non-trivial solution of the homogeneous equation (19). According to the Lemma $\mu_{*}^{0} \in C^{0, p}\left(\Gamma^{1}\right) \cap C^{0}\left(\Gamma^{2}\right)$ for $p=\min \left\{\lambda, \frac{1}{2}\right\}$. Therefore the function $\mu^{0}=\mu_{*}^{0} Q^{-1} \in$ $C_{\frac{1}{2}}^{p}\left(\Gamma^{1}\right) \cap C^{0}\left(\Gamma^{2}\right)$ converts the homogeneous equations (13), (18) into identities. Using the homogeneous identity (18) we check that $\mu^{0}$ satisfies conditions (5). Besides, acting on the homogeneous identity (18) with a singular operator with the kernel $(s-t)^{-1}$ we find that $\mu^{0}$ satisfies the homogeneous equation (14). Consequently, $\mu^{0}$ satisfies the homogeneous equation (12). On the basis of Theorem 2, $W\left[0, \mu^{0}\right](x)=w\left[\mu^{0}\right](x)$ is a solution of the homogeneous problem U. According to Theorem $1, w\left[\mu^{0}\right](x) \equiv 0$ on $\mathcal{D} \backslash \Gamma^{1}$. Using the limit formulas for tangent derivatives of an angular potential [3], we obtain

$$
\lim _{x \rightarrow x(s) \in\left(\Gamma^{1}\right)+} \frac{\partial}{\partial \tau_{x}} w\left[\mu^{0}\right](x)-\lim _{x \rightarrow x(s) \in\left(\Gamma^{1}\right)} \frac{\partial}{\partial \tau_{x}} w\left[\mu^{0}\right](x)=\mu^{0}(s) \equiv 0 \quad\left(s \in \Gamma^{1}\right) .
$$

Hence, $w\left[\mu^{0}\right](x)=w_{2}\left[\mu^{0}\right](x) \equiv 0$ on $\mathcal{D}$ and $\mu^{0}$ satisfies the homogeneous equation

$$
-\frac{1}{2} \mu^{0}(s)+\frac{i}{4} \int_{\Gamma^{2}} \mu^{0}(\sigma) \frac{\partial}{\partial \mathbf{n}_{x}} \mathcal{H}_{0}^{(1)}(\beta|x(s)-y(\sigma)|) d \sigma=0 \quad\left(s \in \Gamma^{2}\right) .
$$

This Fredholm equation is well-known in classical mathematical physics. We arrive at it when solving the Neumann problem for the Helmholtz equation (2) $)_{a}$ in the domain $\mathcal{D}$ by the single layer potential. It is well-known [3] that the equation (20) has only the trivial solution $\mu^{0}(s) \equiv 0$ in $C^{0}\left(\Gamma^{2}\right)$. This is true for both the internal and external domain $\mathcal{D}$.

Consequently, $\mu^{0}(s) \equiv 0$ and $\mu_{*}^{0}(s)=\mu^{0}(s) Q^{-1}(s) \equiv 0$ on $\Gamma$ and we arrive at a contradiction to the assumption that $\mu_{*}^{0}$ is a non-trivial solution of the homogeneous equation (19). Thus, the homogeneous Fredholm equation (19) has only a trivial solution in $C^{0}(\Gamma)$.

We have proved the following assertion.

Theorem 3. If $\Gamma^{1} \in C^{2, \lambda}$ and $\Gamma^{2} \in C^{1, \lambda} \quad(\lambda \in(0,1])$, then (19) is a Fredholm equation of the second kind in the space $C^{0}(\Gamma)$. Moreover, equation (19) has a unique. solution $\mu_{*} \in C^{0}(\Gamma)$ for any $\Phi \in C^{0}(\Gamma)$.

As a consequence of Theorem 3 and the Lemma we obtain the following corollary.

Corollary. If $\Gamma^{1} \in C^{2, \lambda}, \Gamma^{2} \in C^{1, \lambda}(\lambda \in(0,1])$ and $\Phi \in C^{0, p}\left(\Gamma^{1}\right) \cap C^{0}\left(\Gamma^{2}\right)$, where $p=\min \left\{\lambda, \frac{1}{2}\right\}$, then the unique solution of equation $(19)$ in $C^{0}(\Gamma)$, ensured by Theorem 3 , belongs to $C^{0, p}\left(\Gamma^{1}\right) \cap C^{0}\left(\Gamma^{2}\right)$.

We recall that $\Phi$ belongs to the class of smoothness required in the Corollary if $f \in C^{0, \lambda}(\Gamma)$. As mentioned above, if $\mu_{*} \in C^{0, p}\left(\Gamma^{1}\right) \cap C^{0}\left(\Gamma^{2}\right)$ is a solution of the equation (19), then $\mu=\mu_{*} Q^{-1} \in \dot{C}_{\frac{1}{2}}^{p}\left(\Gamma^{1}\right) \cap C^{0}\left(\Gamma^{2}\right)$ is a solution of the system (12), (5). We obtain the following statement. 
Proposition. If $\Gamma^{1} \in C^{2, \lambda}, \Gamma^{2} \in C^{1, \lambda}$ and $f \in C^{0, \lambda}(\Gamma) \quad(\lambda \in(0,1])$, then the system of equations (12), (5) has a solution $\mu \in C_{\frac{1}{2}}^{p}\left(\Gamma^{1}\right) \cap C^{0}\left(\Gamma^{2}\right)\left(p=\min \left\{\frac{1}{2}, \lambda\right\}\right)$, which is expressed by the formula $\mu(s)=\mu_{*}(s) Q^{-1}(s)$ where $\mu_{*} \in C^{0, p}\left(\Gamma^{1}\right) \cap C^{0}\left(\Gamma^{2}\right)$ is the unique solution of the Fredholm equation (19) in $C^{0}(\Gamma)$.

We remind that, if conditions (3) hold, then $f \in C^{0, \lambda}(\Gamma)$ and the solution of equations (5), (12) ensured by the Proposition belongs to $C_{\frac{1}{2}}^{p}\left(\Gamma^{1}\right) \cap C^{0, \frac{\lambda}{2}}\left(\Gamma^{2}\right)$. On the basis of Theorem 2 we arrive at the following final result.

Theorem 4. If $\Gamma^{1} \in C^{2, \lambda}$ and $\Gamma^{2} \in C^{1, \lambda}$, and if the conditions (3) hold, then the solution of the problem $\mathbf{U}$ exists and is given by $(7)$, where $\nu=\nu(s)$ is defined in (10) and $\mu=\mu(s)$ is a solution of the equations (12), (5) from $C_{\frac{1}{2}}^{p}\left(\Gamma^{1}\right) \cap C^{0}\left(\Gamma^{2}\right)$ with $p=\min \left\{\frac{1}{2}, \lambda\right\}$ ensured by the Proposition. More precisely, $\mu \in C_{\frac{1}{2}}^{p}\left(\Gamma^{1}\right) \cap C^{0, \frac{\lambda}{2}}\left(\Gamma^{2}\right)$.

It can be checked directly that the solution of the problem $U$ satisfies condition (1) with $\epsilon=-\frac{1}{2}$. Explicit expressions for singularities of the solution gradient at the end-points of the open curves can be easily obtained with the help of formulas presented in [14].

Theorem 4 ensures the existence of a classical solution of the problem $U$ when $\Gamma^{1} \epsilon$ $C^{2, \lambda}, \Gamma^{2} \in C^{1, \lambda}$ and conditions (3) hold. The uniqueness of the classical solution follows from Theorem 1 . On the basis of our consideration we suggest the following scheme for solving the problem U. First, we find the unique solution $\mu_{*}=\mu_{*}(s)$ of the Fredholm equation (19) from $C^{0}(\Gamma)$. This solution automatically belongs to $C^{0, p}\left(\Gamma^{1}\right) \cap C^{0}\left(\Gamma^{2}\right)$ with $p=\min \left\{\lambda, \frac{1}{2}\right\}$. Second, we construct the solution of equations (12), (5) from $C_{\frac{1}{2}}^{p}\left(\Gamma^{1}\right) \cap C^{0}\left(\Gamma^{2}\right)$ by the formula $\mu(s)=\mu_{*}(s) Q^{-1}(s)$. This solution automatically belongs to $C_{\frac{1}{2}}^{p}\left(\Gamma^{1}\right) \cap C^{0, \frac{\lambda}{2}}\left(\Gamma^{2}\right)$. Finally, substituting $\nu(s)$ from $(10)$ and $\mu(s)$ into (7) we obtain the solution of the problem $\mathbf{U}$.

Acknowledgements. The author is thankful to Professor R. Sakamoto for the opportunity to carry out research in Japan. The research was supported by the RFBR grant No. 96-01-01411.

\section{References}

[1] Aesvestas, J. S.: Electromagnetic scattering by perfectly conducting open screens. Univ. of Delaware: Technical Report from March 1977.

[2] Becache, E. and T. Ha Duong: A space-time variational formulation for the boundary integral equation in a 2.D elastic crack problem. Math. Modelling Numer. Anal. 28 (1994), $141-176$.

[3] Colton, D, and R. Kress: Integral Equation Methods in Scattering Theory. New York: John Wiley \& Sons 1983.

[4] Durand, M.: Layer potentials and boundary value problems for the Helmholtz equation in the complement of a thin obstacle. Math. Meth. Appl. Sci. 5 (1983), $389-421$.

[5] Fabes, E., Jodeit, M. and N. Riviere: Potential technique for boundary value problems on $C^{1}$ domains. Acta Math. 141 (1978), $165-186$. 
[6] Gakhov, F. D.: Boundary Value Problems. Oxford: Pergamon Press 1966.

[7] Ha-Duong, T.: On the transient acoustic scattering by flat object. Japan J. Appl. Math. 7 (1990), $489-513$.

[8] Ha-Duong, T.: On the boundary integral equations for the crack opening displacement of flat cracks. Int. Equ. Oper. Theory 15 (1992), $427-453$.

[9] Hayachi, Y.: The Dirichlet problem for the two-dimensional Helmholtz equation for an open boundary. J. Math. Anal. Appl. 44 (1973), 489 - 530.

[10] Hayachi, Y.: Three-dimensional Dirichlet problem for the Helmholtz equation for an open boundary. Proc. Japan Acad. (Ser. A) 53 (1977), 159 - 162.

[11] Jerison, D. and C. Kenig: The Neumann problem on Lipshitz domains. Bull. Amer. Math. Soc. 4 (1981), $203-207$.

[12] Kirsch, A.: Generalized boundary value and control problems for the Helmholtz equation. Postdoc. Thesis. Goettingen (Germany) 1984, 244 pp.

[13] Krutitskii, P. A.: Dirichlet problem for the Helmholtz equation outside cuts in a plane. Comp. Maths. Math. Phys. 34 (1994), 1073 - 1090.

[14] Krutitskii, P. A.: Neumann problem for the Helmholtz equation outside cuts in a plane. Comp. Maths. Math. Phys. 34 (1994), $1421-1431$.

[15] Maul, J.: Potential method for treating mixed problems in plane elasticity. Uspekhi Mehaniki 6 (1983), $89-98$.

[16] Muskhelishvili, N. I.: Singular Integral Equations. Groningen: Noordhoff 1972.

[17] Nazarchuk, Z. T.: Numerical Analysis of the Diffraction of Waves by Cylindrical Structures (in Russian). Kiev: Naukova Dumka 1989.

[18] Panasyuk, V. V., Savruk, M. P. and Z. T. Nazarchuk: The Method of Singular Integral Equations in Two-Dimensional Diffraction Problems (in Russian). Kiev: Naukova Dumka 1984.

[19] Petersdorff, T. V.: Boundary integral equations for mixed Dirichlet, Neumann and transmission problems. Math. Meth. Appl. Sci. 11 (1989), $185-213$.

[20] Stephan, E. P.: Boundary integral equation for screen problem in $\mathbb{R}^{3}$. Int. Equ. Oper. Theory 10 (1987), $263-283$.

[21] Tuchkin, Yu. A.: Scattering of waves by an unclosed cylindrical screen of an arbitrary profile with Neumann boundary condition (in Russian). Dokl. Akad. Nauk SSSR 293 (1987), $343-345$.

[22] Verchota, G.: Layer potentials and boundary value problems for Laplace equation in Lipshitz domains. J. Funct. Anal. 59 (1984), $572-611$.

[23] Vladimirov, V. S.: Equations of Mathematical Physics. New York: Marcel Dekker 1971.

[24] Zakharov, Ye. V. and I. V. Sobyanina: One-dimensional integro-differential equations of problems of diffraction by screens (in Russian). Zh. Vychisl. Matem. Mat. Fiz. 26 (1986), $632-636$. 